\title{
Reconstrucción de articulación interfalángica proximal del meñique con injerto autólogo osteoarticular y tendinoso de dedo del pie
}

\author{
Reconstruction of the proximal interphalangeal joint of the fifth \\ digit with an osteoarticular and tendinous autograft from toe
}

\section{Efraín Farías Cisneros, ${ }^{\star}$ Cinthya Vargas Zavala, ${ }^{\ddagger}$ Claudia Arroyo Berezowsky, ${ }^{*}$ Luis Andrés Vega Anzures ${ }^{\S}$}

\section{RESUMEN}

Las lesiones traumáticas que involucran la articulación interfalángica proximal de los dedos son muy comunes. En ocasiones se acompañan de complicaciones tales como dolor, rigidez y/o artrosis que limitan la función de forma importante. Cuando esto ocurre suelen ser necesarios procedimientos secundarios para la mejoría funcional, especialmente cuando la superficie articular se ve afectada. Se presenta un caso de artrosis postraumática secundaria a fractura articular compleja de la articulación interfalángica proximal del meñique de la mano dominante, tratada con injerto osteoarticular y tendinoso autólogo no vascularizado del tercer dedo del pie izquierdo. El paciente fue evaluado con historia clínica detallada, exploración física y estudios de imagen. Con un seguimiento de 18 meses postoperatorios. A un año y medio de la cirugía el injerto se encuentra completamente integrado sin datos de condronecrosis. El paciente presenta limitación en los rangos de movilidad; sin embargo, el dedo es funcional y la satisfacción tras el procedimiento es alta, el sitio de toma de injerto no muestra morbilidad. En pacientes jóvenes con lesiones articulares en quienes otras opciones de reconstrucción no son la mejor opción, el injerto autólogo puede proporcionar larga durabilidad y la menor morbilidad posible.

Palabras clave: Articulación interfalángica proximal, artroplastia de dedo, injerto autólogo osteocondral.

Nivel de evidencia: IV

\begin{abstract}
Traumatic injuries involving the proximal interphalangeal joint of the fingers are common. Occasionally, they are accompanied by complications such as chronic pain, stiffness and/or arthritis. These complications may impair function severely. When this occurs, secondary procedures may be necessary to improve function, especially when the articular surface is involved. We present a clinical case of posttraumatic arthritis after a complex intra-articular fracture of the proximal interphalangeal joint of the small finger of the dominant hand, treated with a nonvascularized autologous osteoarticular and tendinous graft from the left third toe. The patient was evaluated with detailed medical history, physical examination and imaging studies. Follow-up to 18 months after surgery. One year and a half after the surgery the graft is completely healed, without signs of chondronecrosis. The patient has restricted range of motion; however, the finger is functional and the satisfaction after the procedure is high. The donor site has recovered and shows no morbidity. In young patients, with joint injuries, when other reconstructive options are not suitable, autologous graft may provide long durability and low morbidity.
\end{abstract}

Keywords: Proximal interphalangeal joint, finger arthroplasty, osteochondral autograft.

Level of evidence: $I V$
* Cirujano de Mano y Ortopedista.

$\ddagger$ Residente de 4to año de Ortopedia.

$\S$ Anestesiólogo Regional.

Centro Médico ABC

Recibido para publicación: 18/01/2020. Aceptado: 03/03/2020.
Correspondencia: Efraín Farías Cisneros. MD, PhD.

Centro Médico ABC Campus Santa Fe. Av. Carlos Graef Fernández Núm. 154, Torre Central, consultorio 104-105, Col. Tlaxala, 05300, Cuajimalpa, CDMX. Tels: 55966155 / 5596 6178. www.manointegral.com E-mail: drefrain@drefrainfarias.com

Abreviaturas:

IFP = Interfalángica proximal.

$\mathrm{MCF}=$ Metacarpofalángica

IFD = Interfalángica distal. 


\section{INTRODUCCIÓN}

Las fracturas de falange proximal que involucran la articulación interfalángica suelen ser comunes en jóvenes tras un traumatismo durante el desarrollo de algún tipo de actividad física. En los casos de fracturas o fracturas-luxaciones, el tratamiento indicado e inmediato es aquél que permita la recuperación funcional completa y con ello evitar la artrosis postraumática. ${ }^{1}$ En caso de no obtener una reducción anatómica, la articulación puede perder movilidad y presentar dolor persistente, limitando la función. En casos de pérdida ósea y/o condral, o lesiones cutáneas y de tendones extensores asociados, la reconstrucción es un reto mayor. La importancia de un manejo correcto radica en que tanto la articulación interfalángica proximal (IFP) como la articulación metacarpofalángica $(\mathrm{MCF})$ son cruciales para la funcionalidad de la mano. ${ }^{2}$ Las actividades diarias de la mano requieren de al menos $60^{\circ}$ de movilidad en las articulaciones IFP y MCF y de $39^{\circ}$ en la articulación interfalángica distal (IFD). ${ }^{3}$

Tras un tratamiento inicial nulo o inadecuado en el que no se reduce la fractura, no se consigue alineación anatómica o no se restaura la función mecánica del dedo afectado, los esfuerzos deben dirigirse a la reconstrucción articular dañada. En el caso de la articulación IFP existen diversas alternativas de tratamiento cuyos objetivos son: restablecer una articulación funcional, reducir el dolor y conseguir un arco de movilidad funcional. ${ }^{4}$ Las alternativas de tratamiento para lesiones de la articulación IFP incluyen amputación, artrodesis, prótesis, espaciadores y transferencia articular, ya sea no-vascularizada o vascularizada. ${ }^{1,5}$
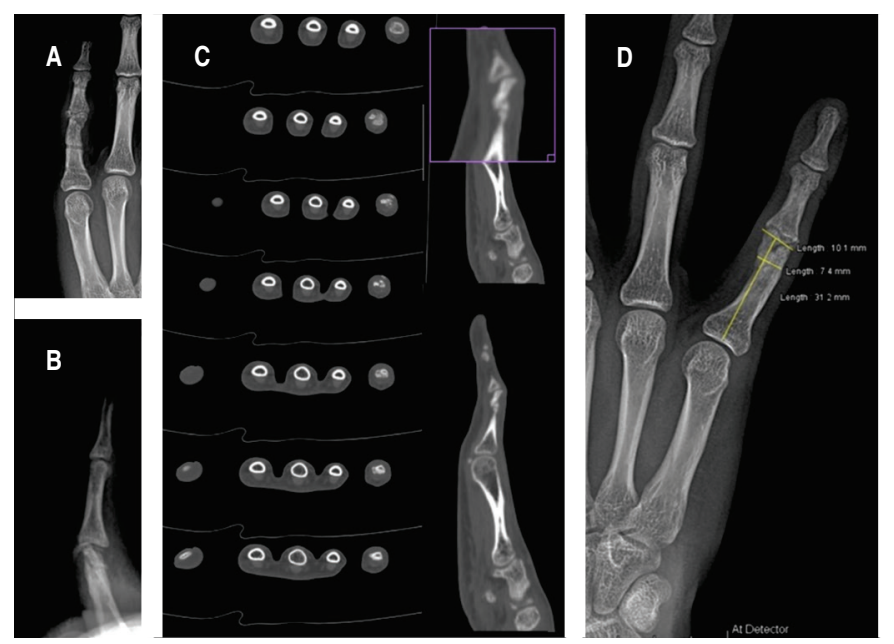
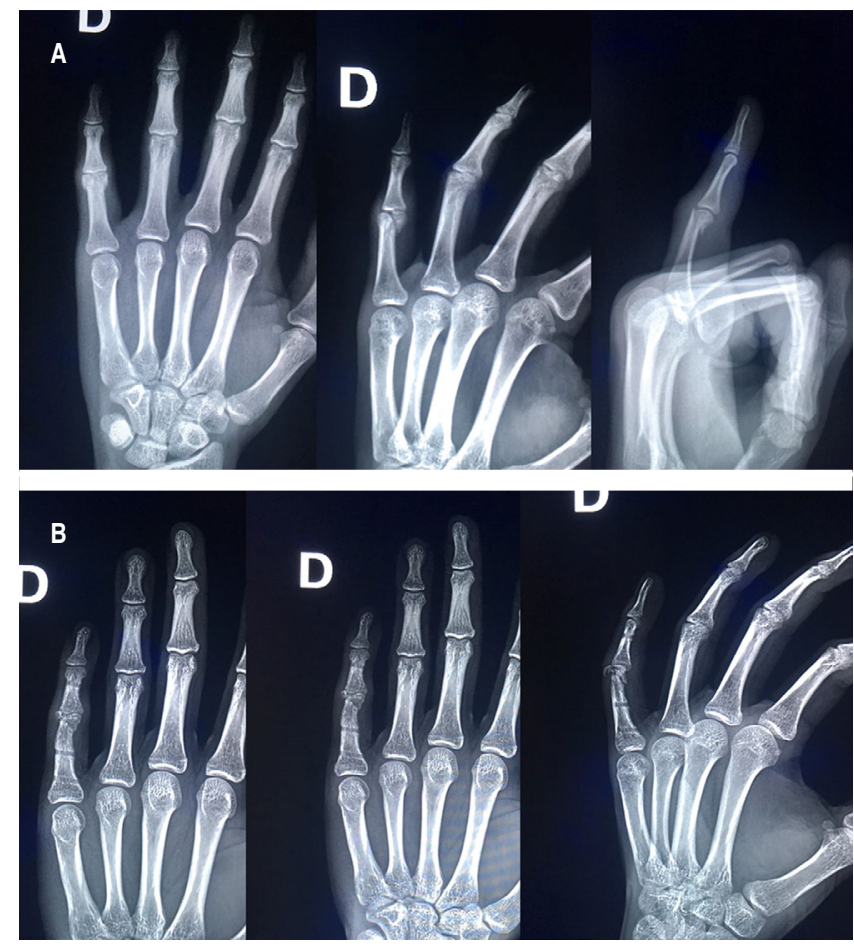

Figura 1: A) Radiografías DP, oblicua y lateral de la mano derecha del día 1. Nótese la fractura articular compleja en forma de " $Y$ » con acortamiento y desplazamiento hacia la placa palmar. La articulación es congruente. B) Radiografías DP y lateral del meñique derecho tras la remoción del fijador externo. Se observa resorción ósea de los fragmentos condilares, así como pérdida del espacio articular de la IFP.

El objetivo de este trabajo es reportar el resultado funcional y radiográfico de un paciente tras el tratamiento de una artrosis postraumática de la articulación IFP del dedo meñique derecho por medio de una

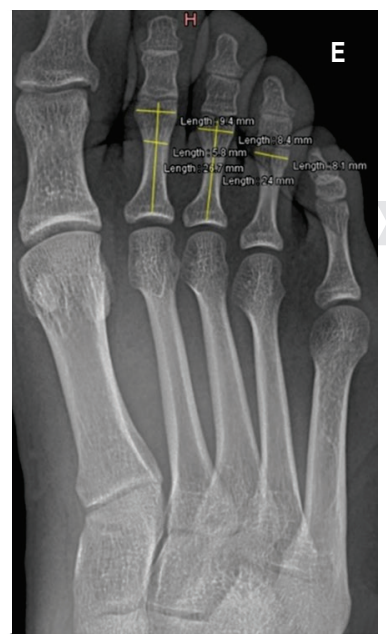

Figura 2:

A y B) La planificación preoperatoria incluyó radiografías simples ortogonales, C) tomografía computarizada, D y E) así como mediciones en radiografías de mano derecha y pie izquierdo en las que se corroboró que la falange donadora más parecida es la proximal del tercer dedo del pie. 

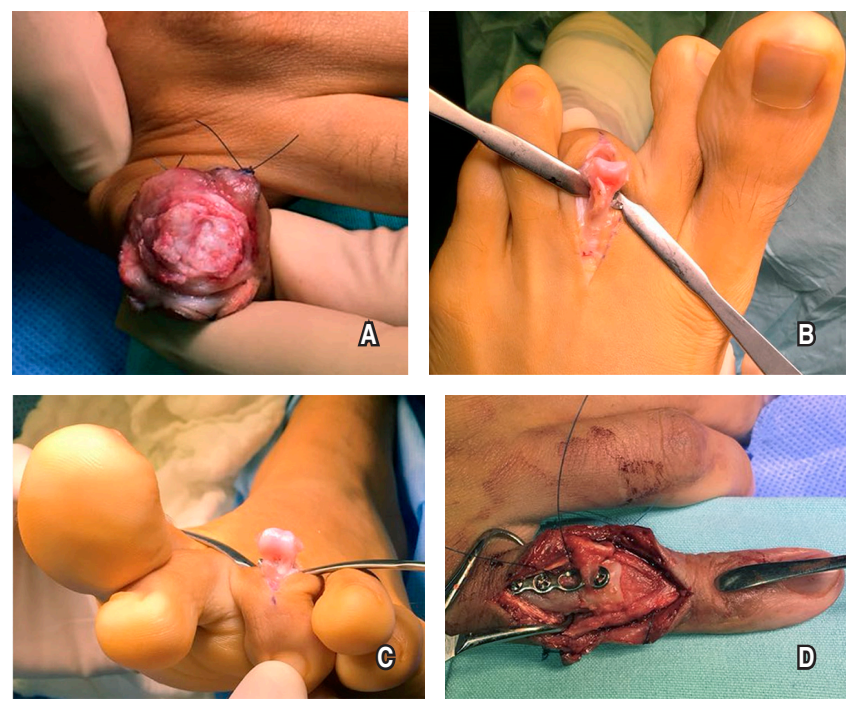

Figura 3: A) Abordaje dorsal a la articulación IFP del meñique derecho, en la que se observa artrosis postraumática. B y C) Exposición del injerto osteocondral de la falange proximal del tercer dedo del pie, incluyendo la bandeleta central del tendón del extensor digitorum longus. Cápsula y ligamentos permanecen en su sitio para realizar la artroplastia por interposición en el sitio donador. D) Imagen transoperatoria con el injerto en su sitio, el colgajo del extensor digitorum longus se encuentra retraído para visualizar el injerto osteocondral bicondíleo.

artroplastia con injerto autólogo del tercer dedo del pie contralateral.

\section{REPORTE DE CASO}

Paciente masculino de 16 años previamente sano, estudiante, quien en octubre de 2017 recibió una contusión directa en el dedo meñique derecho con un balón de básquetbol. Se presentó dolor, deformidad, aumento de volumen y limitación para la movilidad de la articulación IFP. Acudió a urgencias de un hospital privado, las radiografías mostraron una fractura bicondilar, articular compleja, de la cabeza de la falange proximal del dedo meñique derecho (Figura 1A). El tratamiento inicial consistió en inmovilización en extensión de las articulaciones MCF, IFP e IFD del dedo afectado y se envió a consulta externa con ortopedista, quien lo valoró dos semanas después de la fractura. En los siguientes días se le realizó una primera intervención consistente en reducción cerrada y fijación percutánea con dos clavos Kirschner 0.045 para disminuir la articulación y se inmovilizó con una férula en extensión incluyendo la articulación MCF por dos semanas más.
Dos semanas posteriores a la cirugía se tomaron nuevas radiografías de control sin encontrar datos de consolidación. Por tal motivo, se decidió realizar una segunda intervención quirúrgica para retiro de clavillos, reducción abierta y colocación de minifijador externo. Al retirar el fijador externo, cuatro semanas más tarde, el paciente fue enviado a fisioterapia buscando mejorar arcos de movilidad del dedo meñique derecho, ya que presentaba incapacidad para la flexión y extensión de la articulación IFP y dolor en dicha zona (Figura 1B).

Por falta de progreso clínico, en mayo de 2018, es llevado por sus padres con el autor principal, quien a la exploración física dirigida observó el dedo meñique derecho con una actitud en flexión de $20^{\circ}$ de la articulación IFP, además de incapacidad para la flexión y extensión de ésta tanto activa como pasiva. La piel dorsal sobre la IFP mostraba una cicatriz transversal, con abundante fibrosis, además de cuatro cicatrices dorsales puntiformes en los sitios de entrada del fijador externo. Por su parte, la articulación interfalángica distal se encontró en actitud de flexión con incapacidad para la extensión activa. El dedo tenía sensibilidad normal en ambos lados del pulpejo y las arterias digitales se encontraban permeables.
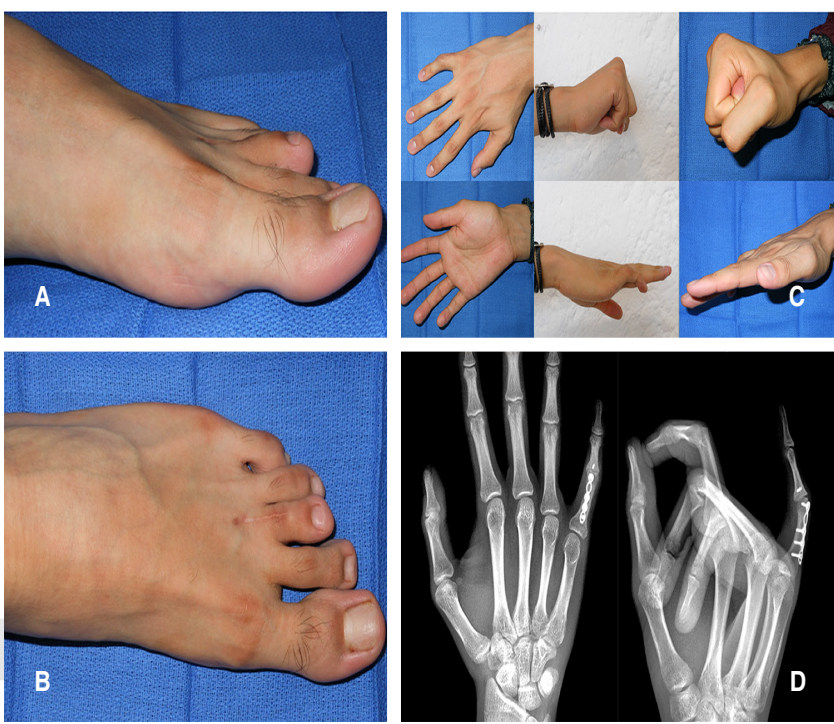

Figura 4: A y B) Fotografías finales del aspecto del pie izquierdo, el paciente realiza actividades de la vida diaria, recreativas y deportivas sin complicaciones. C) Aspecto clínico de la mano derecha. Funcionalmente, el paciente logra hacer puño completo, con punta-palma del meñique completa. En el aspecto estético, el paciente está satisfecho con la alineación del dedo. D) Radiografías DP y oblicua de la mano derecha que muestra osteointegración completa del injerto y preservación del espacio articular, con congruencia articular. 
Nuevos estudios radiológicos del dedo meñique derecho mostraron secuelas de fractura articular bicondílea y pérdida de la anatomía condilar de la cabeza de la falange proximal. Además, se observó una mala unión con desplazamiento hacia cubital y dorsal, con esclerosis subcondral. La articulación IFP mostró disminución del espacio articular.

Se confirmó el diagnóstico de artrosis postraumática de IFP de dedo meñique derecho con anquilosis y lesión del mecanismo extensor. Se comentó con el paciente y sus padres las opciones de manejo que, debido a la presencia de daño condral, incluyeron artrodesis y diversos tipos de artroplastia. Finalmente, se optó por el manejo que se consideró más adecuado para la edad y actividad del paciente, tratando de preservar la mayor movilidad posible del dedo durante el mayor lapso posible. Se planificó una artroplastia IFP con injerto autólogo no vascularizado. Se realizó una tomografía computarizada para caracterizar con mayor detalle la lesión y una radiografía del pie izquierdo en la que se observó que el tercer dedo era el que mejor coincidía con las medidas del dedo meñique, por lo que se utilizaría para la artroplastia (Figura 2).

\section{Técnica quirúrgica}

Bajo bloqueos regionales torácico y lumbar más sedación se colocó al paciente en decúbito supino con la extremidad torácica derecha sobre una mesa de mano y la extremidad pélvica izquierda con un bulto sobre la hemipelvis. En primera instancia se realizó un abordaje dorsal longitudinal sobre la línea media del meñique derecho cruzando la IFP. Acto seguido, se expuso el mecanismo extensor, el cual se encontraba fibroso y con lesión completa de la bandeleta central. Posteriormente, se realizó una capsuloplastia exponiendo la articulación IFP, así como la deformidad y artrosis de los cóndilos de la falange proximal (Figura 3A). Se resecó la cabeza de la falange proximal justo distal a la inserción de los ligamentos colaterales para preservar la estabilidad articular.

En un segundo tiempo se colocó isquemia neumática tras desangrar por gravedad la extremidad pélvica izquierda. Se realizó un abordaje dorsal longitudinal sobre el tercer dedo para la toma del injerto. Se expuso la cabeza de la falange proximal y se obtuvo el injerto incluyendo cartílago articular, epífisis y metáfisis junto con un segmento de la bandeleta central del aparato extensor proveniente del extensor digitorum longus con un poco más de longitud para la sobreposición con la bandeleta central del mecanismo extensor del dedo receptor (Figura $3 B$ y $C$ ). Una vez obtenido el injerto se realizó una artroplastia por interposición de la articulación IFP del tercer dedo del pie con la placa plantar, cerrando la articulación con la cápsula dorsal. Se reconstruyó el mecanismo extensor y se suturó la piel, colocando un vendaje oclusivo.

Por último, en el dedo meñique se colocó el injerto autólogo, el cual se fijó con una placa anatómica premoldeada de $1.6 \mathrm{~mm}$ de cinco orificios con cuatro tornillos, uno cortical y tres bloqueados (Figura 3D). Posteriormente, se realizó la reconstrucción de la banda central con un ancla de $1.3 \mathrm{~mm}$ de diámetro, dirigida a la base falange media, para sujetar el extremo distal del mecanismo extensor del injerto, proximalmente se reparó la bandeleta central receptora al suturarla al extremo proximal del injerto extensor. Se suturó el resto de la herida y se cubrió con un apósito estéril.

El paciente inició movimientos activos y pasivos controlados del meñique, incluyendo la IFP, 48 horas posteriores a la cirugía. Se utilizó una férula dorsal en extensión de la IFP, con las articulaciones MCF e IFD libres durante las noches por seis semanas. Inició terapia ocupacional a la quinta semana de postquirúrgico.

\section{RESULTADOS}

A los 18 meses de postoperado el paciente se presenta asintomático, retomando su vida normal, satisfecho por el aspecto y la función del dedo meñique. El sitio donador también se encuentra asintomático (Figura 4 A y B). A la exploración física el paciente puede tocar con la punta del dedo la palma con arcos de movilidad activos de la articulación IFP de $30^{\circ}$, con extensión máxima de $-30^{\circ}$ y flexión máxima de $60^{\circ}$. La articulación IFD presenta un rezago extensor de $-20^{\circ}$ y flexión de $70^{\circ}$. La articulación metacarpofalángica evidencia una hiperextensión activa de $15^{\circ}$ y flexión completa (Figura $4 C$ ). Las radiografías finales muestran el injerto consolidado, material de osteosíntesis sin datos de aflojamiento y no muestran datos de condronecrosis (Figura 4D).

\section{DISCUSIÓN}

A través de los años se han ido buscando diversas alternativas que devuelvan de la mejor manera posible la funcionalidad a las articulaciones interfalángicas cuando exista una lesión que comprometa la articulación. Con el objetivo de disminuir el dolor y preservar o aumentar los arcos de movilidad se han descrito múltiples opciones reconstructivas antes de considerar una artrodesis, incluyendo prótesis e in- 
An Med (Mex) 2020; 65 (1): 72-78

jertos. Este caso clínico permite demostrar el injerto autólogo no vascularizado como una de las opciones quirúrgicas con resultados satisfactorios.

La artrodesis y amputación sacrifican la articulación, aunque permanecen como opciones viables en algunas situaciones. En la artrodesis se conserva el dedo, se sacrifica la movilidad y esto aporta alivio del dolor, estabilidad, durabilidad y fuerza. La amputación se indica poco en daños aislados, pero se considera en mutilación de un solo dedo cuando las lesiones asociadas son complejas y comprometen supervivencia y función. ${ }^{1,2}$

Las prótesis y los espaciadores teóricamente tienen ciertas ventajas que pueden hacer recomendable su uso; sin embargo, aún presentan problemas de estabilidad, durabilidad y movilidad, lo que limita su aplicación, en especial en pacientes jóvenes ${ }^{1,4}$ como en el caso que nos ocupa. Por otro lado, en presencia de una gran pérdida ósea, el espaciador es imposible de colocar, ya que requiere una cantidad suficiente de hueso para su estabilidad. Además, las prótesis provocan osteólisis que genera su hundimiento y puede llevar a una rigidez en extensión progresiva no funcional que podría evitarse con una artrodesis adecuada. ${ }^{1}$ Los arcos de movilidad obtenidos con la prótesis de silicón llegan a un máximo de $35^{\circ},{ }^{4}$ que va disminuyendo con el tiempo, principalmente en la articulación IFP. ${ }^{1}$ De las prótesis que prometen una mejor funcionalidad son las de pirocarbono; sin embargo, el rango de movilidad recuperado es similar al obtenido con técnicas de artroplastia de tejidos blandos, además se han observado mayores complicaciones que con las de silicón, tales como infecciones, luxaciones y cirugía de revisión, además de la falta de osteointegración. ${ }^{4}$ Otra condición indispensable para indicar el uso de prótesis o espaciadores en la IFP es la presencia de un mecanismo extensor funcional, ${ }^{1}$ que no era el caso de nuestro paciente. En general, las prótesis disponibles en la actualidad deben mejorar en diseño, biocompatibilidad y demostrar una supervivencia larga que justifique su uso en pacientes jóvenes. ${ }^{4}$

Otra razón por la que el uso de prótesis no es lo ideal es el hecho de que las articulaciones digitales son complejas, por lo tanto, el reemplazo articular biológico es una alternativa razonable. ${ }^{1,6-9} \mathrm{El}$ reemplazo biológico comprende diversos tipos, los injertos articulares pericondrales, interposición de varios materiales, aloinjertos y, por último, los autoinjertos que pueden ser: transferencias articulares parciales, articulares completas, vascularizadas o no vascularizadas.

La interposición con injertos pericondrales, pericardio liofilizado o placa palmar se han dejado de usar por tener poco respaldo de la literatura y resultados anecdóticos ${ }^{1}$ generalmente malos. Una práctica considerada aceptable con antelación era la procuración de una articulación homodigital o heterodigital sana para reconstruir la IFP utilizando las estructuras de un dedo amputado o de la IFD del mismo dedo, más la artrodesis subsiguiente del dedo donador. Sin embargo, la necesidad de sacrificar la función de un dedo o articulación sana de la misma mano sobrepasa el beneficio funcional esperado.

En cuanto a los injertos vascularizados procedentes de los dedos menores del pie a la mano, una serie de reportes publicados en 2006 demuestra resultados exitosos. ${ }^{7}$ Esta alternativa es muy útil para falanges, ya que el fragmento a sustituir suele ser muy pequeño y un injerto de cresta iliaca o de radio sobrepasa el tamaño requerido. Otras ventajas son su resistencia a la infección, por lo que se usan para tratar defectos con infección previa y que permiten la curación precoz. No obstante, técnicamente es un reto mantener vascularizados segmentos óseos tan pequeños y el riesgo de trombosis aumenta. Las indicaciones de usar un injerto vascularizado incluyen la presencia de defectos óseos largos, defectos pequeños asociados con defecto de tejidos blandos, infección en un lecho cicatricial deficiente o la necesidad de cartílago. Si bien, los injertos óseos vascularizados de falanges del pie tienen el tamaño ideal para su uso en los defectos de la mano, la confiabilidad del injerto óseo vascularizado disminuye con su tamaño. ${ }^{7}$ La edad es un factor pronóstico importante, ya que en niños hay ausencia de deterioro radiológico, así como la persistencia del disco de crecimiento. ${ }^{1}$ Además, mientras menor es el injerto, mayor es la dificultad de incluir sus vasos nutrientes. En el caso de necesidad de incluir tejidos blandos específicos, se limitan aún más los sitios donadores posibles. Finalmente, los vasos sanguíneos involucrados son en extremo pequeños, por lo que este tipo de cirugías deben reservarse a equipos de microcirugía con amplia práctica y experiencia. ${ }^{7} \mathrm{La}$ mayoría de los defectos óseos pueden ser tratados con injerto óseo no vascularizado, aunque por lo general son sólo córtico-esponjosos o esponjosos.

El injerto autólogo no vascularizado para la reconstrucción de la articulación IFP es una buena alternativa de tratamiento, se han descrito diversas modalidades que han dado buenos resultados funcionales y salvamento de la articulación. En el caso de injertos autólogos parciales, al restaurar la congruencia articular y la estabilidad ósea se permite la rehabilitación inmediata con movimiento poten- 
cial temprano. ${ }^{9-11}$ Dentro de las condiciones para un trasplante osteocondral exitoso se requiere un rápido restablecimiento del flujo sanguíneo o de la perfusión sinovial para evitar condronecrosis y con ello, permitir la supervivencia y función articular. ${ }^{1}$ De acuerdo con Gaul, ${ }^{5}$ para lograr lo anterior se debe restaurar la anatomía normal de la articulación. Uno de los pasos cruciales de la cirugía es obtener el tamaño correcto del injerto, el cual debe ser lo más cercano a la pieza que se va a sustituir para poder realizar una articulación congruente y evitar presión excesiva sobre el cartílago injertado. Para ello la selección del sitio donador es uno de los aspectos más importantes a considerar durante la planeación quirúrgica. ${ }^{12} \mathrm{Se}$ realizó un estudio para analizar la morfología de la falange proximal de los dedos menores, en el cual se demostró que el tercer dedo del pie, además de tener baja morbilidad, presenta un tamaño correcto para poderse usar como injerto en la articulación IFP, lo anterior fue comprobado durante nuestra planificación quirúrgica. ${ }^{13}$

La artroplastia IFP con injerto autólogo no vascularizado que se utilizó en este caso, permitió salvar la articulación, restaurar la congruencia articular y mejorar el aspecto clínico del dedo. Funcionalmente, aún con la debilidad del mecanismo extensor, el paciente recuperó un arco de movimiento activo de $30^{\circ}$, lo que representa la capacidad de asir objetos y hacer puño al poder llevar la punta del meñique a la palma. A nivel radiológico se consiguió consolidación ósea y no hay datos indirectos de resorción condral. Una minucia técnica fue el tamaño del injerto, pues al tomar el injerto de extensor y requerir fijación con estabilidad angular es posible que el injerto haya quedado ligeramente largo, ocasionando ligera flexión de la IFP. Creemos que la edad del paciente, la ausencia de comorbilidades, consumo de tabaco y alcohol influyeron en el resultado obtenido. Desafortunadamente, es casi imposible realizar ensayos clínicos controlados en este tipo de pacientes; sin embargo, nuestro resultado es alentador, ya que confirma lo descrito en otras publicaciones con respecto al salvamento articular con injertos osteocondrales no vascularizados, lo que disminuye la complejidad técnica y los costos.

\section{CONCLUSIÓN}

Las lesiones postraumáticas de la IFP presuponen una morbilidad importante, por ello deben ser tratadas desde el inicio por un cirujano de mano con experiencia. Aunque el tratamiento ideal de las secuelas incluye restablecer una articulación funcio- nal, reducir el dolor y conseguir un arco de movilidad funcional, en ocasiones muchos de los procedimientos logran sólo uno de los objetivos previos. Es por esto que ninguna de las técnicas de reconstrucción articular disponibles ha demostrado ser la ideal. En el caso del injerto autólogo, la similitud de la IFP de los dedos de la mano con la IFP de los ortejos provee una solución práctica y de fácil acceso. ${ }^{5}$

Tras realizar una evaluación de cada una de las opciones terapéuticas para este caso clínico se decidió llevar a cabo una artroplastia IFP con injerto autólogo no vascularizado que proporcionó un resultado satisfactorio para el paciente y sus padres. Se demostró que esta técnica es una excelente alternativa de tratamiento para casos de artrosis postraumática en pacientes jóvenes. A diferencia del injerto vascularizado ésta no tiene tantas dificultades técnicas y es más económica para el paciente. En este caso, los resultados a 18 meses han sido muy favorables, recuperando rangos de movilidad funcionales y un alto grado de satisfacción.

\section{BIBLIOGRAFÍA}

1. Foucher G, Khouri A. Finger joint reconstruction after mutilation of the hand. Acta Orthop Belg. 1997; 63 (3): 144155 .

2. Kotwal PP, Nagaraj C, Gupta V. Vascularised joint transfer in the management of recurrent giant cell tumour of the second metacarpal. J Hand Surg Eur Vol. 2008; 33E (3): 314-316.

3. Hume MC, Gellman H, McKellop H, Brumfield RH Jr. Functional range of motion of the joints of the hand. J Hand Surg Am. 1990; 15 (2): 240-243.

4. Adkinson JM, Chung KC. Advances in small joint arthroplasty of the hand. Plast Reconstr Surg. 2014; 134 (6): 1260-1268.

5. Gaul JS. Articular fractures of the proximal interphalangeal joint with missing elements: Repair with partial toe joint osteochondral autografts. J Hand Surg Am. 1999; 24 (1): 78-85.

6. Zaidenberg EE, Farias-Cisneros E, Miller R, Zaidenberg CR. Metacarpal aneurysmal bone cyst en bloc resection reconstructed with osteoarticular allograft. J Hand Surg Am. 2019; 44 (5): 425.e1-425.e5.

7. del Piñal F, García-Bernal FJ, Delgado J, Sanmartín M, Regalado J, Cagigal L. Vascularized bone blocks from the toe phalanx to solve complex intercalated defects in the fingers. J Hand Surg Am. 2006; 31 (7): 1075-1082.

8. Ellis PR, Hanna D, Tsai TM. Vascularized single toe joint transfer to the hand. J Hand Surg Am. 1991; 16 (1): 160-168.

9. Seifman MA, Thomas DJ, Ek EW. Osteochondral graft for unicondylar resurfacing of finger interphalangeal joints. ANZ J Surg. 2018; 88 (1-2): E95-E96.

10. Williams RM, Kiefhaber TR, Sommerkamp TG, Stern PJ. Treatment of unstable dorsal proximal interphalangeal fracture/dislocations using a hemi-hamate autograft. J Hand Surg Am. 2003; 28 (5): 856-865.

11. Boulas HJ. Autograft replacement of small joint defects in the hand. Clin Orthop Relat Res. 1996; (327): 63-71. 
An Med (Mex) 2020; 65 (1): 72-78

12. Dumont C, Albus G, Kubein-Meesenburg D, Fanghänel J, Stürmer KM, Nägerl H. Morphology of the interphalangeal joint surface and its functional relevance. J Hand Surg Am. 2008; 33 (1): 9-18.
13. Hendry JM, Mainprize J, McMillan C, Binhammer P. Structural comparison of the finger proximal interphalangeal joint surfaces and those of the third toe: suitability for joint reconstruction. J Hand Surg Am. 2011; 36 (6): 1022-1027. 